\title{
Standard Electrocardiographic Data of Peccaries (Tayassu tajacu)
}

\author{
André Braga de Souza', Renan Paraguassu de Sá Rodrigues', Gerson Tavares Pessoa', Andrezza Braga Soares da \\ Silva', Laecio da Silva Moura', Francisco das Chagas Araújo Sousa' ${ }^{2}$, Kássio Vieira Macedo ${ }^{3}$ \& Flávio Ribeiro Alves ${ }^{4}$
}

\begin{abstract}
Background: Peccaries (Tayassu tajacu, Linnaeus, 1758) are wild suiformes that belong to the Tayassuidae family. Electrocardiography is an important technique for cardiovascular evaluation. Analysis of various intervals, segments, complexes and waveforms of electrocardiographic (ECG) traces aids in the diagnosis of cardiac alterations and in the differentiation of congenital and acquired heart diseases from physiological cases. However, in wild animal medicine, the various patterns of normality and the evaluation of electrical traces associated with heart disease have not yet been sufficiently elucidated. The purpose of this study was to characterize the electrocardiographic (ECG) traces of peccaries sedated using ketamine and xylazine.

Materials, Methods \& Results: Fourteen healthy adult animals that were subjected to digital ECG examination were used. Animals with evidence of systemic diseases, cardiovascular abnormalities (murmurs or arrhythmias), or any degree of valve insufficiency observed on echocardiogram and animals that exhibited excessive stress during the examination were excluded from the study. All animals presented with a normal sinus rhythm. A combination of $15 \mathrm{mg} / \mathrm{kg}$ of ketamine hydrochloride and $3 \mathrm{mg} / \mathrm{kg}$ of midazolam maleate was applied intramuscularly for chemical immobilization. The animals were manipulated after $15 \mathrm{~min}$, when the onset of the anaesthetic effect was verified, for a duration of $45 \mathrm{~min}$, and no reinforcement dose was necessary to complete the electrocardiographic examination. No significant differences were observed in the P-wave duration, $\mathrm{PR}$ interval and QT interval between genders $(P>0.05)$. No significant differences were found between the amplitudes of the $\mathrm{P}$ and $\mathrm{R}$ waves between males and females $(P>0.05)$. The observed $P$ waves were small, monophasic and positive. The QRS complex was positive in the DI, DII, DIII, aVF, V4 and V10 derivations and negative in the aVR, aVL, V1 and V2 derivations. In $71 \%$ of the animals, the T wave showed negative polarity in the DI, DII, DIII, aVL, aVF, and V10 derivations and positive polarity in the aVR, V1, V2 and V4 derivations. The ST segment was isoelectric in $100 \%$ of the animals. GraphPad Prism 7 (La Jolla, CA, USA) software was used to analyze the data, with non-parametric tests used to test for differences in the variables between the sexes. In these tests, a $P$-value of 0.05 was considered to indicate statistical significance.

Discussion: Although studies on the cardiac electrophysiology of wild animals have previously shown good results for several species, this is the first study concerning the standardization ECG traces for peccaries. However, due to the wild nature of these animals, their manipulation for handling and data collection purposes is only feasible under chemical containment, although other studies have used non-anaesthetized agoutis. It is not known to what extent these results may have been influenced by the effects of stress. Drugs used for this function may have direct effects on cardiac function. Therefore, the presumed normal ECG values, as well as the recognition of changes due to drug or iatrogenic interactions, are of fundamental importance. This protocol provided high-quality anaesthetized peccary ECG traces, allowing reliable measurements of waves and intervals and assessment of the cardiac rhythm and heart rate. The surface registry digital ECG recording technique used with chemical containment allowed good monitoring and rapid acquisition and was well tolerated by the animals.
\end{abstract}

Keywords: wild animals, cardiology, anesthesia, Tayassuidae, heart. 


\section{INTRODUCTION}

Peccaries (Tayassu tajacu, Linnaeus, 1758) are wild suiformes that belong to the Tayassuidae family and have similarities to domestic pigs [2,4,17,25]. When treated improperly, peccaries often exhibit clinical changes compatible with stress, and cases of death are not rare $[3,19]$. However, basic information about the cardiovascular physiology of wild species is lacking, which indicates the importance of the standardization of values of normal cardiovascular function for this group of animals to perform adequate monitoring as well as the selection of the ideal drug for this purpose [1].

Among the drugs of choice, ketamine has shown satisfactory results for the containment of wild animals due to its high lethal dose [7,13,14]. However, it should be used with other drugs, such as alpha-2 agonists or benzodiazepines [15,28]. In peccaries, this combination has shown good results due to the elimination of stressors [3].

Electrocardiography is an important technique for cardiovascular evaluation, specifically for the identification and qualification of arrhythmias. However, in wild animal medicine, the various patterns of normality and the evaluation of electrical traces associated with heart disease have not yet been sufficiently elucidated $[11,23]$ and descriptive studies on the ECG trace profile of these animals, obtained under sedation, have not been reported to date.

Due to the necessity of chemical restraint and the importance of knowing the cardiovascular effects for correct use of anaesthetic protocols in medical clinics for wild animals, this study aimed to characterize the ECG traces of chemically contained peccaries using a combination of ketamine and xylazine.

\section{MATERIALS AND METHODS}

\section{Animals}

Fourteen healthy peccaries (Tayassu tajacu) were obtained from the Center for the Study and Preservation of Wild Animals - NEPAS, Federal University of Piauí CCA/UFPI, Teresina, PI. The animals were divided in two groups, the first consisting of 8 males and the second consisting of 6 females; all animals were adults with an average age of four years and weights between 17.5 and $19.3 \mathrm{~kg}$. These animals were fed twice daily with a commercial diet for pigs, containing $18 \%$ crude protein and various vegetables as a source of fibre, energy, minerals and water, and had ad libitum access to the feed.
Subjects with an unremarkable clinical history and physical examination were included in the study. The cardiovascular and respiratory systems were inspected by auscultation of the heart valves and lung fields. The time of capillary perfusion and the diagnosis of dehydration were also evaluated by hemodynamic investigation. Echocardiography examination was performed by a single, experienced examiner ( $\mathrm{Ph} . \mathrm{D}$. in diagnostic imaging). Animals with evidence of cardiovascular abnormalities (murmurs or arrhythmias), or any degree of valve insufficiency observed on echocardiogram were excluded from the study.

\section{Inclusion and exclusion criteria}

Subjects with an unremarkable clinical history and physical examination were included in the study. The cardiovascular and respiratory systems were inspected by auscultation of the heart valves and lung fields. The time of capillary perfusion and the diagnosis of dehydration were also evaluated by hemodynamic investigation. Systemic infectious processes were evaluated by palpating the peripheral lymph nodes (submandibular, cervical, and popliteal), abdominal palpation, and temperature measurement. In addition, all of the animals were subjected to blood, biochemical, and electrolyte tests. Animals with evidence of systemic diseases, cardiovascular abnormalities (murmurs or arrhythmias), or any degree of valve insufficiency observed on echocardiogram and animals that exhibited excessive stress during the examination were excluded from the study.

\section{Anesthetic protocol}

The animals were fasted from food for $12 \mathrm{~h}$ and water for $3 \mathrm{~h}$ and captured in their enclosures with hand nets. A combination of $15 \mathrm{mg} / \mathrm{kg}$ of ketamine hydrochloride $^{1}$ and $3 \mathrm{mg} / \mathrm{kg}$ of midazolam maleate ${ }^{2}$ was applied intramuscularly for chemical immobilization. The animals were manipulated after $15 \mathrm{~min}$, when the onset of the anaesthetic effect was verified, for a duration of $45 \mathrm{~min}$, and no reinforcement dose was necessary to complete the electrocardiographic examination.

\section{Computerized electrocardiography}

To perform the tests, the animals were positioned in the right lateral decubitus position, with the parallel limbs extended and perpendicular to the long axis of the body, on a table with a rubber insulating surface to avoid interference.

The ECG exams were performed using a computerized method for digital veterinary electrocar- 
diography (Electrocardiogram Acquisition Module for the Computer (ECG-PC Windows 95 version) ${ }^{3}$ with 10 derivations (DI, DII, DIII, aVL, aVF, aVR, V1, V2, V4 V10), consisting of an electronic circuit connected externally to a laptop and software installed on the hard disk of a computer. After the examination, the results for each animal were analysed, and the wave measurements were made on the computer. The heart rate (HR) was measured in the ECG trace.

The electrodes were specifically distributed throughout the body of the animal in standardized combinations, according to the methodology described by Tilley [30], to yield standard ECG derivations, which were obtained through the potential difference between the electrodes. After automatic capture performed by the software, the measurements of the durations and amplitudes of the complete P-QRS-T were performed manually.

The right and left thoracic electrodes were positioned above the olecranon in the caudal aspect, and the right and left pelvic electrodes were positioned above the patellar ligaments in the cranial aspect of each limb. The speed used for the registers was 25 $\mathrm{mm} / \mathrm{s}$, with a voltage calibration of $1 \mathrm{~cm}$ for each millivolt $(1 \mathrm{mV}=1 \mathrm{~cm})$.

The aspects evaluated included the HR, duration in milliseconds (ms) and amplitude in $\mathrm{mV}$ of the $\mathrm{P}$ wave, PR interval, QRS complex, amplitude of the $\mathrm{R}$ wave, ST segment levelling, QT interval, corrected QT interval, QT/RR ratio and T wave polarity and amplitude, which were all analysed in bipolar derivation II (DII). The measurement of the cardiac electrical axis in degrees $\left(^{\circ}\right)$ of the QRS was made considering the algebraic sum of the QRS deflections in the DI and DIII derivations, as previously described by Tilley [30].

\section{Statistical analysis}

GraphPad Prism 7 (La Jolla, CA, USA) software was used to analyze the data, with non-parametric Wilcoxon-Mann-Whitney (Mann-Whitney U) tests used to test for differences in the variables between the sexes. The Spearman rank correlation coefficient was used to assess dependence. In these tests, a $P$-value of 0.05 was considered to indicate statistical significance.

\section{RESULTS}

Table 1 shows the mean and standard deviation of the results for $\mathrm{P}$ wave duration and amplitude, QRS complex duration, QT interval, corrected QT interval,
PR interval, $\mathrm{R}$ and $\mathrm{T}$ wave amplitudes for male and females. Table 2 presents the mean and standard deviation of the HR values in beats per minute (bpm), weight in kilograms $(\mathrm{kg})$ and age in years of the male and female peccaries studied here. The QT/RR ratio showed poor correlation $(\mathrm{r}=-170, P=0.561)$. Identical letters in the same column indicate that no significant differences were observed $(P>0.05)$ for the studied variables when males and females were compared.

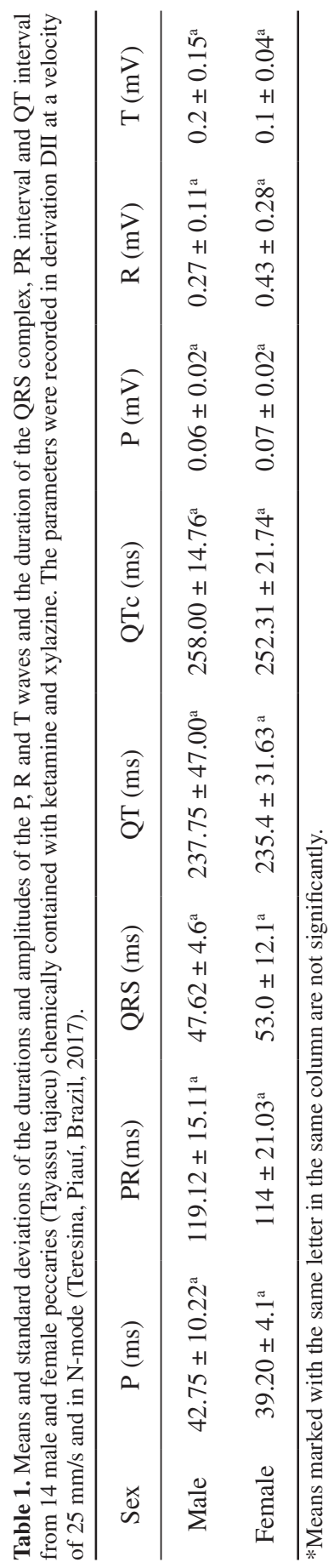


Table 2. Means and standard deviations of the heart rate values in beats per minute (bpm), weights ( $\mathrm{kg}$ ) and ages of 14 male and female peccaries (Tayassu tajacu) chemically contained with ketamine and xylazine. The parameters were recorded in derivation DII at a velocity of $25 \mathrm{~mm} / \mathrm{s}$ and in N-mode (Teresina, Piauí, Brazil, 2017).

\begin{tabular}{cccc}
\hline Sex & HR $(\mathrm{bpm})$ & Weight $(\mathrm{kg})$ & Age (Years) \\
\hline Male & $104.5 \pm 16.0^{\mathrm{a}}$ & $18.4 \pm 0.18^{\mathrm{a}}$ & $4.0 \pm 1^{\mathrm{a}}$ \\
Female & $94.4 \pm 23.21^{\mathrm{a}}$ & $17.9 \pm 0.28^{\mathrm{a}}$ & $4.3 \pm 1^{\mathrm{a}}$ \\
\hline
\end{tabular}

*Means marked with the same letter in the same column are not significantly.

Normal sinus rhythms were found in all study animals (Figure 1). One animal from the male group presented five premature ventricular complexes (PVC) throughout the examination. The animal was replaced by an animal that did not show changes during the examination to maintain homogeneity of the sample. The animal who manifested the ectopic beats was monitored and appropriately treated with an antiarrhythmic drug of choice (Sotalol@ $2.0 \mathrm{mg} / \mathrm{kg} \mathrm{IV}) 4$, with complete resolution of the arrhythmia 3 min after intravenous infusion. The protocol provided high quality ECG traces of anaesthetized peccaries with reliable waves and interval measurements, allowing evaluation of the HR and frequency. Difficulties in the measurements due to breathing artefacts, muscle tremors or poorly captured complexes did not occur in any examination.

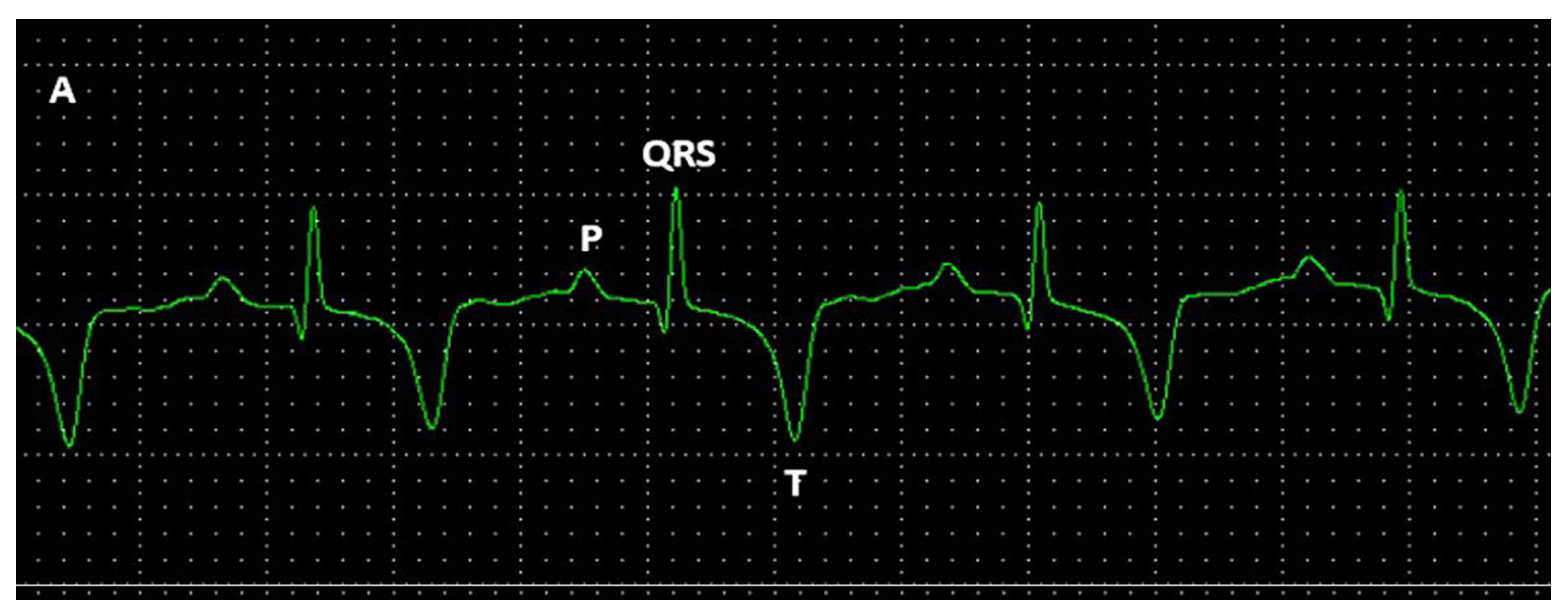

B

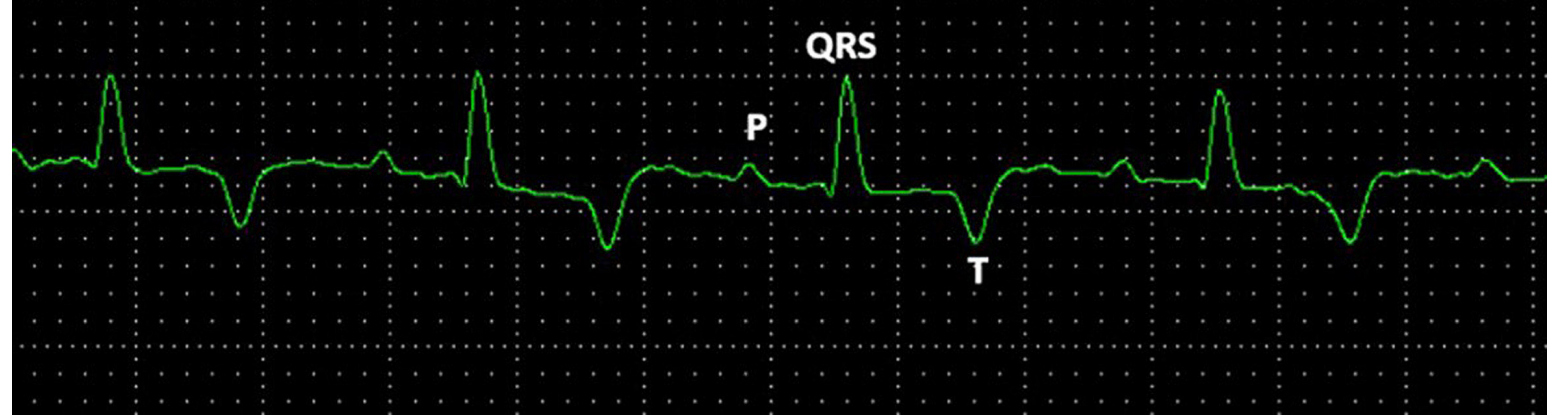

Figure 1. Normal digital electrocardiographic tracing recorded in $25 \mathrm{~mm} / \mathrm{s}$ from healthy adult peccaries (Tayassu tajacu) chemically contained with ketamine and xylazine. A- Sinus rhythm, P wave, QRS complex and T wave (positive polarity) observed in males. B- Sinus rhythm, P wave, QRS complex and T wave (positive polarity) observed in females.

Regarding the duration parameters (measured in ms), no significant differences were observed in the $\mathrm{P}$ wave duration, PR interval and QT interval between genders. For the amplitude parameters, no significant differences were found in the amplitude of the $\mathrm{P}$ and $\mathrm{R}$ waves between males and females. The observed $\mathrm{P}$ waves were small, monophasic and positive in the DI, DII, aVL, aVF, V2 and V4 derivations and negative in 
the DIII, AVR, V1 and V10 derivations for males and females. The QRS complex was positive in the DI, DII, DIII, aVF, V4 and V10 derivations and negative in the aVR, aVL, V1 and V2 derivations (Figure 2).

In 10 animals, the $\mathrm{T}$ wave showed negative polarity in the DI, DII, DIII, aVL, aVF, and V10 leads and positive polarity in the aVR, V1 (rV2), V2 and V4 derivations, and no significant difference was observed between the genders regarding the amplitude of this wave. The ST segment was isoelectric for all animals, in both the male and female groups, without the presence of depression or elevation (Figure 2).

The HR mean, tended to be higher in males, however, did not present significant statistical differences compared to that of females.

The cardiac electrical axis (EEC) showed variations between the individuals. In 10 animals the cardiac electrical axis varied between $7^{\circ}$ and $115^{\circ}$ (Figure $3 \mathrm{~A}$ ), while in 4 animals, between $-7^{\circ}$ and $-150^{\circ}$ (Figure 3B).

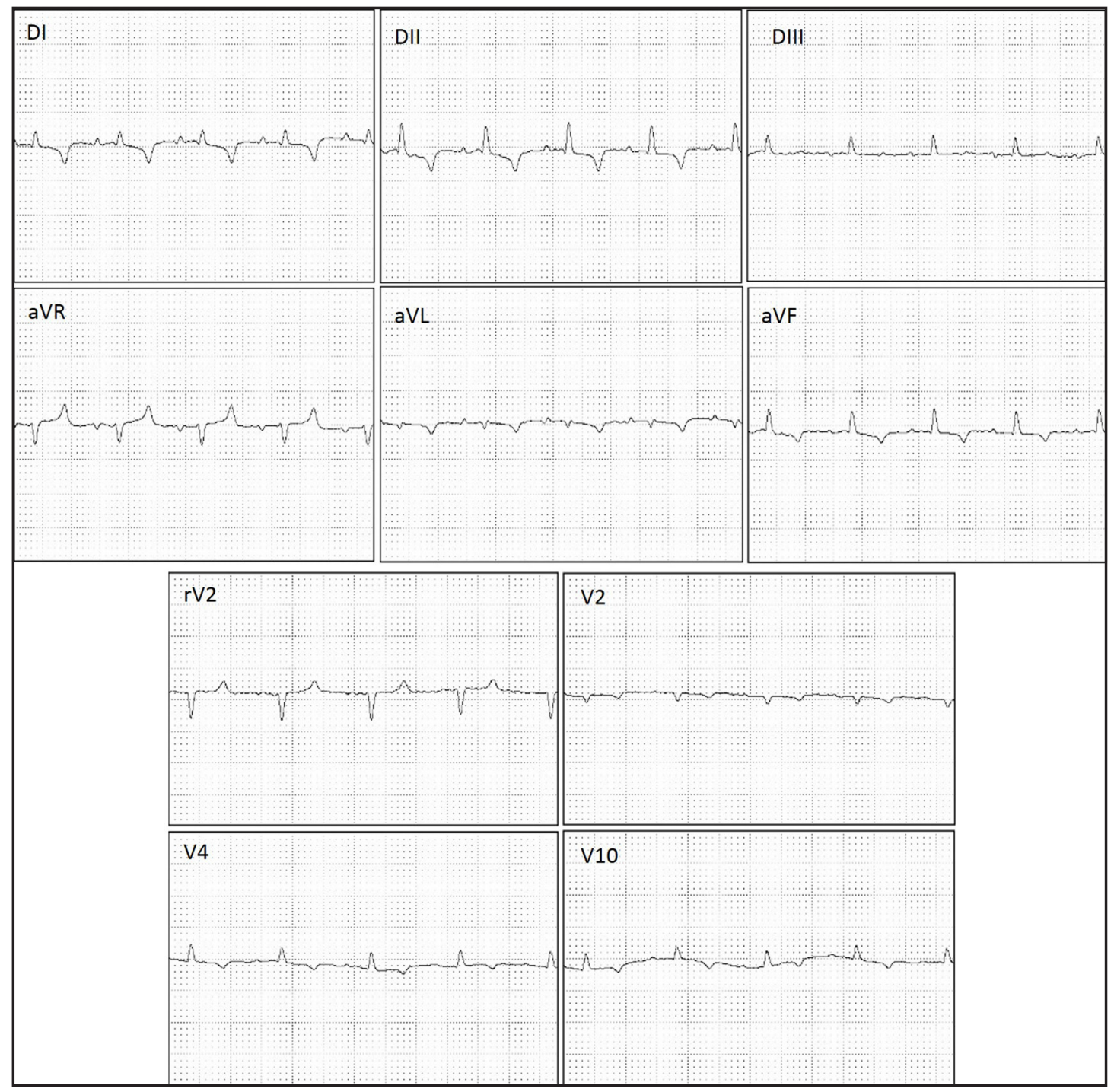

Figure 2. Normal digital electrocardiographic tracing recorded in $25 \mathrm{~mm} / \mathrm{s}$ and from healthy peccaries (Tayassu tajacu) chemically contained with ketamine and xylazine. Note the QRS complex was positive in the DI, DII, DIII, aVF, V4 and V10 derivations and negative in the aVR, aVL, V1 and V2 derivations. 


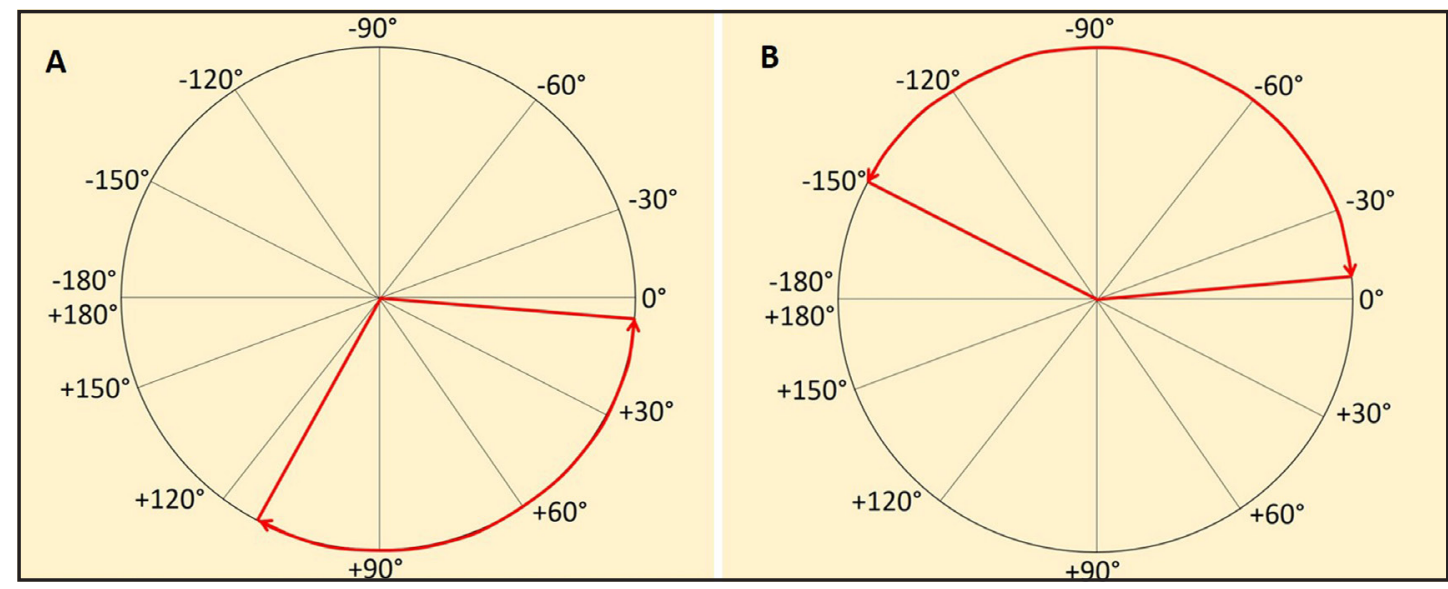

Figure 3. The cardiac electrical axis (EEC) showed variations between the individuals from healthy peccaries (Tayassu tajacu) chemically contained with ketamine and xylazine. A- In 10 animals the cardiac electrical axis varied between $7^{\circ}$ and $115^{\circ}$. B- In 4 animals, between $-7^{\circ}$ and $-150^{\circ}$.

\section{DISCUSSION}

Although studies on the cardiac electrophysiology of wild animals have previously shown good results for several species [13], this is the first study concerning the standardization ECG traces for peccaries (Tayassu tajacu). However, due to the wild nature of these animals, their manipulation for handling and data collection purposes is only feasible under chemical containment, although other studies have used non-anaesthetized agoutis [8]. It is not known to what extent these results may have been influenced by the effects of stress [2]. Drugs used for this function may have direct effects on cardiac function. Therefore, the presumed normal ECG values, as well as the recognition of changes due to drug or iatrogenic interactions, are of fundamental importance [7].

The HR values found in this study were similar to the findings for pigs (Sus scrofa domestica), guinea pigs and peccaries $[3,32,33]$. Although they belong to different families, domestic pigs and peccaries are physically similar [4], justifying the similarity in HR values among these species. Factors such as age, body position, functional capacity and subspecies differences may also affect HR values. The standardized positioning, the similarity of characteristics between males and females and the similarity of the ages of the animals may explain the lack of significant differences between males and females in relation to the HR [20]. Only a few publications have described the normal ECG of pig breeds. Specifically, fours study of conventional and miniature pigs obtained varied ECG values and patterns, possibly related to differences in the breeds, ages, and body weight.
The morphology of the ECG traces measured in the DII derivation of peccaries, since the patterns of measurement and analysis of QRS morphology were best established in this derivation [30]. Despite a few references to ECG patterns, in pigs and Göttingen minipigs was observed QRS measurements (mean duration of $36 \mathrm{~ms}$ and amplitude of $0.9 \mathrm{mV}$ ) and QT interval $(252 \mathrm{~ms})$ similar to those found in this study, although we performed our evaluations in anesthetized animals [23]. Similarly, comparing our results with one of the first studies using awake conventional pigs, QRS duration (mean of $37 \mathrm{~ms}$ ) and QT interval (218 ms) values were also concordant with results for anesthetized peccaries [10].

The present study evidenced a great variation of the QRS patterns in the studied derivations. This variation has also been reported in pigs [10,32] and in small ruminants [21]. These studies attributed this variability of wave deflections to changes in cardiac topography relative to the chest, as well as differences in the ventricular activation mechanism. As observed in horses, cattle, pigs and sheep, ventricular activation seems to occur in two "fronts" of depolarization that begins at the apex of the left ventricle, with terminal depolarization in the middle third and basilar of the interventricular septum, showing an apicobasilar direction, where the left ventricle is activated simultaneously in a subepicardial to epicardial direction. The QT/RR ratio showed a weak correlation, although in other species of ungulates, such as the equine, a significantly strong relation was found [26]. A human study has shown that the QT/RR ratio exhibits a high degree of 
intersubjective variability and may present a linear pattern for some healthy individuals and non-linear for others [27]. In a more in-depth analysis it is believed that QT/RR adaptation may depend on the complex interaction of individual ion channels that maintain the potential for action of ventricular myocytes [24].

The $\mathrm{P}$ wave was positive in the DII derivation in all animals, ranging from 39.2 to $42.75 \mathrm{~ms}$ in duration. These findings were similar to those observed in conventional minipigs and pigs which was within the range considered normal $[10,32]$. The PR interval ranged from 114 to $119.12 \mathrm{~ms}$. Similarly, it was described a PR interval varying between $88-101 \mathrm{~ms}$ in minipigs. The statistical similarities between males and females have also been reported previously on conventional swine and minipigs. Allied to this, in this experiment the anaesthetic protocol did not result in variations in vagal tonus, which may cause slight variations in this interval $[10,22]$.

The $\mathrm{T}$ wave did not show great variability in its shape and polarity, unlike the findings found in some species [9]. Although some studies have suggested that the position of the electrode in the patients thorax may affect T-wave polarity [10], standardization of the position of the electrodes used in this experiment [30] did not show variability in T-wave polarity, which was also observed in Goettingen minipigs [22,32].

An inverted polarity of the $\mathrm{T}$ wave was observed in the studied peccaries. Likewise, this configuration was observed in dogs and horses [11]. A morphological study of the cardiac conduction system has shown that, in ungulates, Purkinje fibers that reach the endocardium can penetrate the myocardium. As in dogs, the $\mathrm{T}$ wave in the peccaries may develop a unidirectional transmural gradient, the inner wall is more negative than the outer wall, leading one to believe that the repolarization sequence occurs from the epicardium to the endocardium [29].

It is important to emphasize that, unlike our study, all previous studies were performed with animals awake, without the action of sedatives or anesthetics. Nevertheless, the anesthetic association employed did not produce cardiac electrical conduction alterations (alteration of repolarization), showing QT and QTc intervals similar to those reported for pigs, although xylazine can also trigger heart-related alterations, such as arrhythmias and an atrioventricular block [9,16,].

The $\mathrm{T}$ wave of females in this experiment corresponded to approximately $23 \%$ of the amplitude of the previous $\mathrm{R}$ wave, whereas for males deeper $\mathrm{T}$ waves ( $>25 \%$ of $\mathrm{R}$ wave amplitude) were produced. This relationship is widely studied in dogs, cats and humans, and normal T-wave values are considered to be approximately $25 \%$ to $35 \%$ of the $\mathrm{R}$ wave value in these species. If these percentages are higher, myocardial hypoxia or electrolyte imbalances may be suspected [30]. Male peccaries appear to have had greater anesthetic influence when compared to females. Myocardial ischaemia is more common and better tolerated in animals than in humans and may be due to factors that lead to an increased HR or the use of drugs such as ketamine, as described in studies with primates [5] and dogs [6].

The QT interval ranged from 235.4 to 237.75 , with no significant difference between genderswhich was similar to that found for dogs, conventional swine and minipigs [30]. Variations of the QT interval may be caused by changes in the HR or due to varying degrees of anaesthetic depth. Widening or narrowing of this range is associated with electrolyte imbalances, conduction disturbances, and hypothermia. However, all the peccaries were normothermic during the examinations and did not present clinical signs of dehydration, and the values measured for this interval were likely normal for the species.

The electric axis was a highly variable parameter among all animals of the experiment, as has been observed in several species of monkeys $[5,16,18,27,31]$, as well as in conventional pigs and minipigs $[32,33]$.

The present study aimed at the preliminary acquisition of electrocardiographic values for peccaries. To the best of our knowledge, this is the first work that demonstrates the cardiac electrical behavior of this species after anesthesia. We recognize as a limitation the reduced sample of animals used in this study. In addition to this, it is known that the anesthetic effect provides some interference in the cardiovascular activity of these animals. Despite this, due to the hostility of the species, in addition to excessive stress, which in some cases may have fatal repercussions, these animals can only be managed under chemical containment, and although this happens frequently in our routine with wild animals, there was still no information on the cardiovascular response of these animals under anesthesia. Thus, the study model took into account the most commonly used anesthetic association in 
the NEPPAS-UFPI, at the Wildlife Screening Centers (CETAS) and Zoos of the Northeast of Brazil, for the containment of this species. Another point of difficulty would be the scarcity of scientific literature on nearby species, such as the swine, that allows discussion under the subject in a consistent manner. However, we believe that from an initial perspective, these results may be of great value during the clinical and surgical management of this species.

\section{CONCLUSION}

The surface registry digital ECG recording technique with chemical containment allowed good monitoring and rapid acquisition and was well tolerated by the animals; significant ECG interference was not triggered by the metabolic effect of the anaesthetic protocol. This allowed the reliable acquisition of data and the establishment of ECG parameters in peccaries sedated with ketamine and xylazine.

\section{MANUFACTURERS}

${ }^{1}$ König Brasil Ltda. São Paulo, SP, Brazil.

${ }^{2}$ Roche Brasil S/A. Jaraguá, RJ, Brazil.

${ }^{3}$ Brazilian Electronic Technology - TEB. São Paulo, SP, Brazil.

${ }^{4}$ Merk S/A. São Paulo, SP, Brazil.

Acknowledgments. The authors thank the Diagnosis by Imaging Sector of the University Veterinary Hospital, Federal University of Piauí (UFPI) and the Nucleus for Wild Animal Research and Preservation of the UFPI for making the animals available. They also thank the Coordenação de Aperfeiçoamento de Pessoal de Nível Superior (CAPES) for the doctoral grant.

Ethical approval. The protocols used in this study were approved by the Committee on Ethics in Animal Experimentation - CEAA/UFPI (No. 0.47/2015) and authorized by the Ministry of the Environment, through the System of Authorization and Information of Biodiversity - SISBIO of the Brazilian Institute of the Environment and Renewable Natural Resources - IBAMA $\left(\mathrm{N}^{\circ} \cdot 48113-1\right)$.

Declaration of interest. The authors report no conflicts of interest. The authors alone are responsible for the content and writing of paper.

\section{REFERENCES}

1 Azevedo C.S., Lima M.F.F., Silva V.C.A., Young R.J. \& Rodrigues M. 2012. Visitor influence on the behavior of captive greater rheas (Rheidae aves). Journal of Applied Animal Welfare Science. 15(2): 113-125.

2 Batista J.S., Bezerra F.S.B., Agra E.G.D., Calado E.B., Godói R.M., Rodrigues C.N.F., Nunes F.C.R. \& Blanco B.S. 2009. Efeitos da contenção física e química sobre os parâmetros indicadores de estresse em catetos (Tayassu tajacu). Acta Veterinaria Brasilica. 3(2): 92-97.

3 Batista J.S., Bezerra F.S.B., Lira R.A., Orpinelli S.R.T., Dias C.E.V. \& Oliveira A.F. 2008. Peccary stress syndrome (Tayassu tajacu) submitted to capture and containment at different times in the morning in Mossoró, RN. Ciência Animal Brasileira. 9(1): 170-176.

4 Benirshchke K. 1974. Quest for the giant peccary: the chaco revisited. Zoonozis 25: 364-372.

5 Capriglione L.G.A., Soresini G.C.G., Fuchs T., Sant'Anna N.T., Fam A.L.D., Pimpão C.T. \& Sarraff-Lopes A.P. 2013. Electrocardiographic evaluation of capuchin monkeys (Sapajus apella) under chemical containment with midazolam and propofol. Ciências Agrárias. 34(6): 3801-3810.

6 Clark D.M., Martin R.A. \& Short C.A. 1982. Cardiopulmonary responses to xylazine/ketamine anesthesia in dog. Journal of American Animal Hospital Association. 18(5): 815-821.

7 Diniz A.N., Pessoa G.T., Moura L.S., Sanches M.P., Rodrigues R.P.S., Sousa F.C.A., Ambrósio C.A. \& Alves F.R. 2016. Computerized electrocardiogram in agoutis (Dasyprocta prymnolopha, Wagler 1831) anesthetized with ketamine and midazolam. Pesquisa Veterinária Brasileira. 37(2): 150-155.

8 Diniz A.N., Silva Júnior J.R., Ambrósio C.E., Sousa J.M., Sousa V.R., Carvalho M.A.M., Nascimento D.M. \& Alves F.R. 2013. Thoracic and heart biometrics of non-anesthetized agouti (Dasyprocta prymnolopha, Wagler, 1831) measured on radiographic images. Pesquisa Veterinária Brasileira. 33(3): 411-416.

9 Dubois M. 1961. On the electrocardiograms of some quadrupeds. Comptes Rendus des Séances de la Société de Biologie et de ses Filiales. 155: 599-602.

10 Dukes T.W. \& Szabuniewicz M. 1969. The electrocardiogram of conventional and miniature swine (Sus scrofa). Canadian Journal Compendium of Medicine. 33(2): 118-127.

11 Felippe P.A.N. 2007. Electrocardiography. In: Cubas Z.S., Silva J.C.R. \& Catão-Dias J.L. (Eds). Textbook of Wild Animals. São Paulo: Roca, pp.920-929. 
12 Geiser D.R. 1990. Chemical restraint and analgesia in the horse. Veterinary Clinics of North American: Equine Practice. 6(3): 495-512.

13 Glomset D.J., Anna T.A., Glomset B.S. \& Iowa D.M. 1940. A morphologic study of the cardiac conduction system in ungulates, dog, and man: Part II: The purkinje system. American Heart Journal. 20: 677-701.

14 Green C.J., Knight J., Precious S. \& Simpkin S. 1981. Ketamine alone and combined with diazepam or xylazine in laboratory animals: a 10 year experience. Laboratory Animals. 15(2): 163-17.

15 Haskins S.C., Farver T.M. \& Patz J.D. 1985. Ketamine in dogs. American Journal of Veterinary Research. 46(9): 1855-1860.

16 Larsson M.H.M.A., Pellegrino A., Oliveira V.M.C., Prada C.S., Fedullo J.D.L. \& Larsson-Junior C.E. 2010. Electrocardiographic parameters of captive tufted capuchins (Cebus apella) under chemical immobilization. Journal Zoo and Wildlife Medicine. 43(4): 715-718.

17 Liva H., Moraes L.F.D., Nogueira-Filho S.L.G. \& Lavorenti A. 1989. Aspects of feeding of peccaries (Tajacu) in captivity. In: Annals of the Paulist Congress of Scientific Initiation (São Paulo, Brazil). pp.1-10.

18 Malhotra V., Pick R., Pick A. \& Glick G. 1975. Electrocardiographic studies in the stumptail macaque (Macaca arctoides). Journal of Electrocardiology. 8(3): 247-251.

19 Mayor P., Guimarães D.A.A., Le Pendu I., Silva J., Jori F. \& López-Béjar M. 2007. Reproductive performance of captive collared peccaries (Tayassu tajacu) in the eastern Amazon. Animal Reproduction Science. 102(1-2): 88-97.

20 Migliaro E.R., Contreras P., Bech S., Etxagibel A., Castro M., Ricca R. \& Vicente K. 2001. Relative influence of age, resting heart rate and sedentary life style in short-term analysis of heart rate variability. Brazilian Journal of Medical and Biology Research. 34(4): 493-500.

21 Mohan N.H., Niyogi D. \& Singh H.N. 2005. Analysis of normal electrocardiograms of Jamunapari goats. Journal of Veterinary Science. 6(4): 295-298.

22 Nahas K., Baneux P. \& Detweiler D. 2002. Electrocardiographic monitoring in the Goettingen minipig. Comparative Medicine 52(3): 258-264.

23 Neto G.B.P., Brunetto M.A., Sousa M.G., Carciofi A.C. \& Camacho A.A. 2010. Effects of weight loss on the cardiac parameters of obese dogs. Pesquisa Veterinária Brasileira. 30(2): 167-171

24 Noble D. \& Tsien R.W. 1969. Outward membrane currents activated in the plateau range of potentials in cardiac Purkinje fibers. Journal of Physiology. 200(1): 205-231.

25 Nowak D.M. \& Paradiso J.L. 1948. Walker's Mammals of the World. Baltimore: The John Hopkins University Press, 1184-1185p.

26 Pelentz T. 1971. Normal electrocardiogram in guinea pigs. Acta Physiologica Polonica. 22(1): 113-121.

27 Schmidt-Nielsen K. 1999. Animal physiology: adaptations and the environment. Santos: Livraria Santos, 600p.

28 Souza A.L.P., Paula V.V., Cavalcante P.H. \& Oliveira M.F. 2008. Effect of premedication with acepromazine or xylazine on the induction of dissociative anesthesia with ketamine and diazepam in peccaries (Tayassu tajacu). Ciência Animal Brasileira. 9(4): 1114-1120.

29 Spach M.S. \& Barr R.C. 1975. Ventricular intramural and epicardial potential distributions during ventricular activation and repolarization in the intact dog. Circulation Research. 37(2): 243-57.

30 Tilley L.P. 1992. Essentials of canine and feline electrocardiography: interpretation and treatment. 3rd edn. Philadelphia: Lea and Febiger, 470p.

31 Toback J.M., Clark J.C. \& Moorman W.J. 1978. The electrocardiogram of Macaca fasicularis. Laboratory Animal Science. 28(2): 182-185.

32 Zhang S.B., Guo K.N., Xie F., Liu Y., Shang H.T. \& Wei H. 2016. Normal Electrocardiogram of Bama Miniature Pigs (Sus scrofa domestica). Journal of American Association of Laboratory Animal Science. 55(2): 152-154.

33 Zeman F.J. \& Wilber C.G. 1965. Some characteristics of the electrocardiogram in guinea pigs. Life Sciences. 4(23): 2269-2274. 